\title{
Evolução do Comprimento Cervical Uterino na Gestação, Avaliado pela Ultra-sonografia Transvaginal
}

\author{
Changes in Cervical Length during Pregnancy \\ Measured by Transvaginal Ultrasound
Reginaldo Antônio de Oliveira Freitas-Júnior, Francisco Mauad-Filho
Geraldo Duarte, Adilson Cunha Ferreira
Ana Karla Monteiro Santana de Oliveira Freitas,
George Dantas de Azevedo

\begin{abstract}
RESUM0
Objetivo: analisar a evolução do comprimento cervical uterino, ao longo da gestação, avaliado por meio da ultra-sonografia transvaginal.

Métodos: foi realizado estudo prospectivo, longitudinal, no qual 82 gestantes higidas foram acompanhadas desde o início da gestação, das quais 49 mantiveram o seguimento até o parto sem complicações da gravidez, sendo examinadas a cada quatro semanas, e agrupadas, conforme a paridade, em nuliparas ou com um ou mais partos anteriores. O comprimento do colo uterino foi avaliado em vista sagital pela ultra-sonografia transvaginal, com a medida linear da distância entre os orificios cervicais interno e externo.

Resultados: as médias de comprimento do colo uterino, bem como os percentis 5, 25, 50, 75 e 95, em função da idade gestacional, não diferiram significativamente entre os grupos estudados ( $p>0.05$ ). No intervalo entre a $20^{a}$ e a $24^{a}$ semana gestacional o comprimento cervical variou entre 28, 35 e 47,2 mm, com os percentis 5, 50 e 95, respectivamente. O comprimento cervical uterino diminuiu progressivamente ao longo da gestação normal, sendo esse encurtamento significativo após a $20^{a}$ semana de gravidez e mais expressivo após a $28^{a}$ semana $(p<0.05)$.

Conclusões: o padrão de comportamento do comprimento cervical uterino parece não diferir entre nuliparas e mulheres com um ou mais partos anteriores. Os valores numéricos da curva de normalidade do comprimento cervical uterino, em função da idade gestacional, refletem a variabilidade perante as características peculiares à amostra estudada, devendo, portanto, ser valorizados os parâmetros estabelecidos para a nossa população.
\end{abstract}

PALAVRAS-CHAVE: Comprimento cervical uterino. Gestação normal. Ultra-sonografia transvaginal.

\section{Introdução}

O comprimento do colo uterino, mensurado pela ultra-sonografia transvaginal, é um dos parâmetros que melhor correlação tem apresen-

Departamento de Ginecologia e Obstetrícia da Faculdade de Medicina de Ribeirão Preto da Universidade de São Paulo - FMRP-USP; Escola de Ultra-sonografia e Reciclagem Médica Ribeirão Preto, Ribeirão Preto-SP.

Correspondência:

Reginaldo Antônio de Oliveira Freitas Júnior

Maternidade Escola Januário Cicco

Av. Nilo Peçanha, 270 - Petrópolis,

59012-300 - Natal - RN

Fone: (84) 202-3402/217 2686 / Fax: (84) 234-5900

e-mail: rafarei@terra.com.br tado com o risco para o parto pré-termo, aliando vantagens como aplicabilidade prática, disponibilidade na grande maioria dos serviços de atenção pré-natal, baixo custo, fidedignidade e boa reprodutibilidade ${ }^{1-4}$.

Apesar dos avanços obtidos com a avaliação ultra-sonográfica transvaginal da cérvice uterina e a caracterização de suas modificações na gravidez, ainda não há consenso sobre o comportamento das alterações no comprimento cervical durante a gravidez. Alguns investigadores sugerem que o comprimento do colo mantém-se relativamente constante até o terceiro trimestre, quando só então passaria a diminuir ${ }^{5}$. Outros, entretanto, descrevem constante diminuição dessa medida, já iniciada a partir do segundo trimestre ${ }^{6,7}$. 
Outro aspecto que suscita discussão entre os autores diz respeito às diferenças entre o comportamento do colo uterino nas nulíparas e naquelas mulheres nas quais a cérvice uterina já passou pelo processo da parturição, havendo desde aqueles que descrevem padrões semelhantes de comportamento cervical uterino entre os dois grupos de pacientes ${ }^{6-8}$ até os que relatam colos mais longos e com maior diâmetro para as multíparas ${ }^{9,10}$.

Todas as discrepâncias descritas a respeito do comportamento do colo uterino gravídico resultam de uma série de fatores, variando desde a técnica ultra-sonográfica empregada, diferenças de parâmetros de ordem racial e populacional, incidência de grávidas com risco aumentado para parto pré-termo na população estudada e, inclusive, a falta de critérios de padronização para as medidas cervicais durante a gestação normal.

Assim, em nosso meio, torna-se prioritário avaliar o comportamento do comprimento do colo uterino ao longo de gestações normais, possibilitando, então, mediante a construção de uma curva de normalidade para tais medidas, estabelecer relações de parâmetros em face das gestações patológicas e, sobretudo, aquelas envolvendo maior risco para o parto pré-termo espontâneo, na nossa população.

\section{Pacientes e Métodos}

Foi realizado estudo longitudinal prospectivo, recrutando-se inicialmente 82 grávidas consideradas clinicamente normais, que se submeteram à avaliação ultra-sonográfica transvaginal do colo uterino no início da gestação e, a partir de então, a intervalos de quatro semanas, até a resolução da gravidez. O estudo foi analisado e aprovado pelo Comitê de Ética em Pesquisa do Hospital das Clínicas da Faculdade de Medicina de Ribeirão Preto - USP.

A idade gestacional foi estabelecida com base na data da última menstruação e confirmada por exame ultra-sonográfico realizado até a $12^{\mathrm{a}}$ semana de gestação, ou por, pelo menos, dois exames ultra-sonográficos concordantes realizados entre a $12^{\text {a }}$ e a $20^{\text {a }}$ semana de gestação.

Constituíram critérios de exclusão: gravidezes múltiplas, doenças maternas associadas ou intercorrentes à gravidez estudada, ameaça de parto pré-termo, gravidezes ultimadas antes da $37^{\mathrm{a}}$ semana, amniorrexe prematura, sindromes hipertensivas, restrição de crescimento intrauterino, diabete melito, doenças infecciosas, doenças e malformações fetais, antecedentes de cirurgia prévia no colo uterino (conização, amputação, cerclagem), antecedentes de amniorrexe prematura ou partos prematuros anteriores e índice de massa corporal (IMC) $<18 \mathrm{~kg} / \mathrm{m}^{2}$ (baixo peso) e IMC $>30$ $\mathrm{kg} / \mathrm{m}^{2}$ (obesidade). À anamnese de seleção, as grávidas estudadas negaram exposição a substâncias tóxicas por vício ou risco ocupacional, como fumo, maconha, cocaína, álcool, chumbo e agrotóxicos.

Para a amostra inicial foram recrutadas 82 pacientes, sendo que, em razão da descontinuidade de comparecimento aos retornos agendados ou em obediência aos critérios de exclusão estabelecidos, 49 casos considerados clinicamente normais mantiveram o seguimento até o final do estudo. As voluntárias foram divididas em dois grupos, conforme a paridade: nuliparas $(n=24)$ e multiparas, representando aquelas com um ou mais partos anteriores $(n=25)$.

Os exames ultra-sonográficos foram realizados utilizando-se um único aparelho, por um único observador, treinado e habilitado segundo os princípios da Fundação de Medicina Fetal - FMF Brasil, conforme Certificado de Competência em Avaliação Cervical, tendo a medição do colo uterino obedecido os passos discutidos adiante.

Antes do procedimento, as pacientes foram orientadas para proceder ao esvaziamento vesical completo. As pacientes foram colocadas em posição ginecológica, em decúbito dorsal, com as pernas abduzidas, permitindo-se gama completa de movimentos durante o exame. O transdutor ultrasonográfico transvaginal, de 5 a $9 \mathrm{MHz}$, foi coberto totalmente com preservativo estéril não lubrificado, contendo pequena quantidade de gel transmissor em seu reservatório e, para facilitar a inserção do transdutor no intróito vaginal, utilizouse gel transmissor sobre sua extremidade para lubrificação. O transdutor foi introduzido em direção ao fórnix vaginal anterior, mantido no terço externo do canal vaginal, de forma a se obter uma vista sagital do colo uterino, sem, no entanto, exercer pressão indevida sobre a cérvice uterina, evitando-se seu conseqüente alongamento.

Procedeu-se à identificação do orificio interno, orifício externo, canal cervical e mucosa endocervical, esta última utilizada para definição precisa do nivel do orificio interno e para construção da imagem do canal cervical em toda sua extensão. O campo visual foi ampliado, de tal forma que o colo uterino ocupasse cerca de $75 \%$ da imagem. Efetuou-se a medida linear da distância entre o orificio interno e o externo, procedendo-se a três medidas e imagens, durante período mínimo aproximado de 3 minutos, registrando-se a melhor e mais curta medida do colo uterino.

Em cada avaliação subseqüente, procedeuse ao exame ultra-sonográfico, aferindo-se os parâmetros biométricos fetais e a adequação do 
crescimento fetal, em adição à avaliação cervical uterina, objeto do estudo.

Para determinação da normalidade dos valores amostrais, nos dois grupos estudados, foi utilizado o teste de Kolmogorov-Smirnov, identificandose que todas as variáveis consideradas apresentaram distribuição normal. As comparações entre os resultados obtidos nos dois grupos foram realizadas por meio dos testes $t$ de Student (para variáveis quantitativas) e $\chi^{2}$ (para variáveis qualitativas).

Para analisar o comportamento do comprimento do colo uterino em função do tempo, foram realizados dois tipos de ajustes, por meio da análise de regressão: (linear e exponencial). Após obtenção das curvas do comprimento cervical uterino para os dois grupos estudados, foi realizada comparação intergrupos por meio do teste da coincidência e comparação entre os diferentes intervalos de idade gestacional, por meio do teste do paralelismo. Para verificação de possíveis correlações entre o comprimento cervical uterino e as demais variáveis consideradas, foi calculado o coeficiente de correlação de Pearson. Em todas as comparações foi adotado nível de significância de $5 \%$.

\section{Resultados}

A média da idade materna para a casuística estudada foi de 23,7 \pm 4,95 anos (variação de 15 a 39 anos). A média para o IMC foi de $21,26 \pm 2,94 \mathrm{k} / \mathrm{m}^{2}$ para as nuliparas e de 23,34 $\pm 5,43 \mathrm{kgm}^{2}$, para as multíparas. A distribuição das pacientes segundo a cor mostrou que, em ambos os grupos, houve predomínio das não brancas. Não houve diferenças significativas quanto às características clinicas e demográficas, entre os dois grupos estudados.

A duração da gestação entre as nulíparas foi de 39,5 $\pm 1,12$ semanas, diferindo significativamente daquela observada para as multiparas, nas quais a duração observada foi de 38,9 $\pm 1,02$ semanas $(\mathrm{p}=0,04)$. Quanto à via de resolução do parto, os grupos nulíparas e multíparas apresentaram proporções semelhantes de partos vaginais $(58,3 \mathrm{e}$ $68 \%$ ) e cesáreas $(41,7$ e $32 \%)$, respectivamente. O peso dos recém-nascidos não diferiu entre os dois grupos considerados, sendo a mediana e a média \pm desvio-padrão, respectivamente, de 3.210 e $3.218,1$ $\pm 403,6$ gramas para as muliparas e 3.350 e 3.320,4 $\pm 365,6$ gramas para as multiparas $(p=0,36)$.

A distribuição das médias do comprimento do colo uterino, ao longo das idades gestacionais avaliadas, não mostrou diferença significativa para os valores obtidos entre os dois grupos do estudo (Tabela 1). Tais valores mostraram-se progressivamente decrescentes, em função do tem- po decorrido, tanto nas avaliações individuais dos grupos, quanto na amostra total das pacientes selecionadas.

Tabela 1 - Média \pm desvio padrão do comprimento do colo uterino em milímetros, em função da idade gestacional.

\begin{tabular}{ccccc}
\hline Idade gestacional & Nulíparas & Multíparas & Total & $\mathbf{p}^{*}$ \\
\hline 12 - 16 semanas & $41,4 \pm 6,9$ & $39,0 \pm 7,4$ & $40,2 \pm 7,2$ & 0,24 \\
16 - 20 semanas & $39,5 \pm 6,1$ & $37,6 \pm 6,2$ & $38,5 \pm 6,2$ & 0,27 \\
20 - 24 semanas & $36,9 \pm 6,1$ & $35,2 \pm 5,0$ & $36,0 \pm 5,6$ & 0,30 \\
24 - 28 semanas & $33,2 \pm 5,0$ & $32,9 \pm 3,6$ & $33,1 \pm 4,3$ & 0,78 \\
28 - 32 semanas & $31,0 \pm 5,0$ & $30,9 \pm 4,0$ & $30,5 \pm 4,5$ & 0,50 \\
32 - 36 semanas & $27,6 \pm 5,0$ & $27,5 \pm 4,3$ & $27,5 \pm 4,6$ & 0,96 \\
$\geq 36$ semanas & $22,9 \pm 6,8$ & $23,1 \pm 5,8$ & $23,0 \pm 6,2$ & 0,90 \\
*teste $t$ de Student. Não houve diferença significativa entre os grupos.
\end{tabular}

*teste $t$ de Student. Não houve diferença significativa entre os grupos.

Sendo assim, foi possivel a construção de uma única representação gráfica para a média \pm desvio-padrão do comprimento do colo uterino nas diversas idades gestacionais avaliadas, ilustrando o comportamento dessa variável em função do tempo, onde se percebe que, a partir do intervalo entre a $20^{a}$ e a $23^{a}$ semana gestacional, o comprimento cervical uterino passou a apresentar diminuição importante (Figura 1).

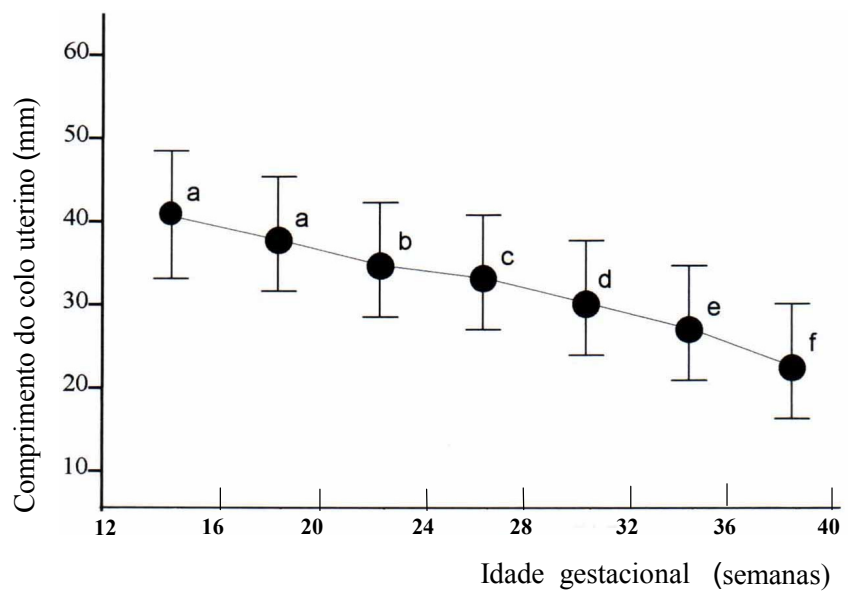

Figura 1- Médias \pm desvios-padrão do comprimento do colo uterino em função da idade gestacional. Letras em comum indicam semelhança estatística. A ausência de letras em comum indica diferença estatisticamente significativa, sendo $a \neq b \neq c \neq d \neq e \neq f(p<0,05$ teste $t$ de Student).

Para determinar o padrão de encurtamento do colo uterino, ao longo das gestações normais observadas, o primeiro passo consistiu em encontrar o modelo que fornecesse o melhor ajuste das medidas obtidas em função do tempo. Com o uso da análise de regressão, foram testados os modelos linear e exponencial, sendo que em ambas as análises as diferenças mostraram-se significati- 
vas $(\mathrm{p}<0.001)$, indicando que há queda dos valores com o passar do tempo, representada pelo coeficiente angular (slope) negativo, em ambos os modelos. E, por ter mostrado ganho superior com a aplicação do ajuste cúbico à regressão exponencial, o modelo exponencial foi o adotado para a construção da curva do comportamento do comprimento do colo uterino, ao longo de gestações normais.

Para a construção da curva de normalidade do comprimento cervical uterino, em função da idade gestacional, foi adotada a distribuição das medidas obtidas segundo percentis. Em face das semelhanças já descritas entre os grupos estudados, em adição à ausência de diferenças significativas entre os valores observados para os referidos percentis entre nuliparas e multíparas ( $p>0,05)$ (Tabela 2), a curva proposta foi construída com base nos resultados do total da amostra em estudo (Figura 2).

Tabela 2 - Valores dos percentis 5, 25, 50, 75, e 95 para o comprimento do colo uterino em milímetros, em função da idade gestacional, obtidos para o grupo nulíparas, grupo multíparas e para o total da amostra.

\begin{tabular}{|c|c|c|c|c|c|c|}
\hline Idade gestacional & Grupos & p5 & p25 & p50 & p75 & p95 \\
\hline \multirow[t]{3}{*}{$12-16$ semanas } & Total & 28,2 & 36,0 & 40,0 & 44,5 & 57 \\
\hline & Nulíparas & 28,1 & 37,3 & 40,5 & 45,0 & 58,1 \\
\hline & Multíparas & 27,2 & 34,5 & 39,0 & 42,0 & 58,2 \\
\hline \multirow[t]{3}{*}{16 - 20 semanas } & Total & 31,0 & 35,0 & 37,0 & 40,0 & 53,1 \\
\hline & Nulíparas & 31,6 & 35,3 & 38,0 & 44,3 & 54,9 \\
\hline & Multíparas & 30,3 & 34,5 & 37,0 & 39,5 & 56,9 \\
\hline \multirow[t]{3}{*}{$20-24$ semanas } & Total & 28,0 & 32,5 & 35,0 & 39,5 & 47,2 \\
\hline & Nulíparas & 28,6 & 33,0 & 35,0 & 40,0 & 53,4 \\
\hline & Multíparas & 26,6 & 32,0 & 34,0 & 38,5 & 47,6 \\
\hline \multirow[t]{3}{*}{$24-28$ semanas } & Total & 26,5 & 30,0 & 33,0 & 36,0 & 42,0 \\
\hline & Nulíparas & 23,9 & 30,3 & 33,0 & 36.0 & 44,9 \\
\hline & Multíparas & 27,3 & 30,0 & 32,0 & 36,0 & 39,4 \\
\hline \multirow[t]{3}{*}{28 - 32 semanas } & Total & 24,5 & 27,5 & 30,0 & 34,0 & 38,9 \\
\hline & Nulíparas & 19,1 & 27.3 & 29,5 & 34,3 & 40,1 \\
\hline & Multíparas & 25,3 & 28,0 & 30,0 & 34,0 & 39,4 \\
\hline \multirow[t]{3}{*}{$32-36$ semanas } & Total & 20,5 & 24,0 & 28,0 & 30,0 & 37,0 \\
\hline & paras & 16,7 & 23,3 & 28,0 & 30,0 & 37,0 \\
\hline & Multíparas & 20,3 & 24,0 & 27,5 & 30,5 & 36 , \\
\hline \multirow[t]{3}{*}{$\geq 36$ semanas } & Total & 10,0 & 20,0 & 23,0 & 27,0 & 36,0 \\
\hline & n & 8.9 & 19,5 & 22.9 & 26,5 & 36 \\
\hline & Multíparas & 10,9 & 20,0 & 23,1 & 27,0 & 34 \\
\hline
\end{tabular}

Em cada intervalo considerado, não houve diferença significativa entre os grupos.

Foram testadas as diferenças relativas à velocidade de encurtamento do colo uterino em todos os segmentos de idade gestacional avaliados. Entretanto, após a $20^{a}$ semana de gestação, quando o encurtamento cervical uterino é mais rápido, a $28^{a}$ semana aparece como ponto de deflexão mais importante para a diminuição do comprimento do colo uterino.

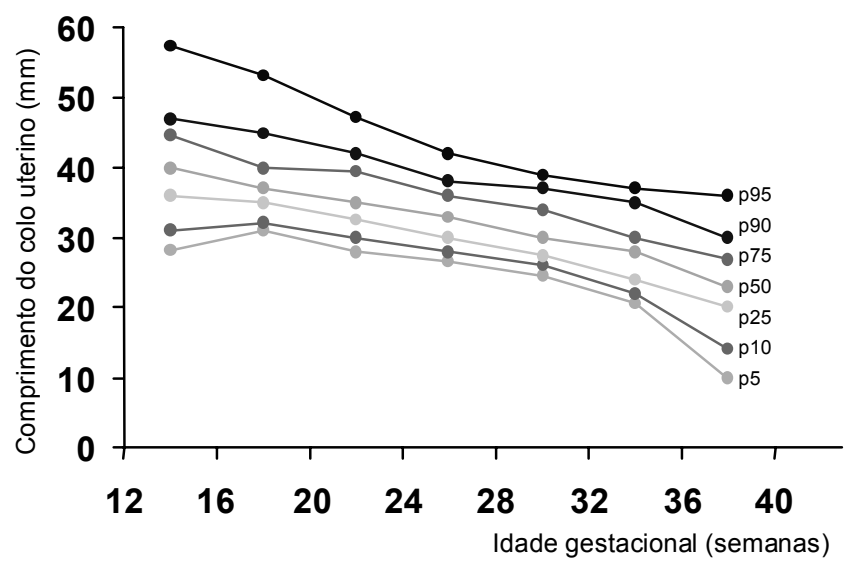

Figura 2 - Curva de normalidade do comprimento do colo uterino na gestação, avaliado pela ultra-sonografia transvaginal. Representação dos percentis 5, 10, 25, 50, 75, 90 e 95.

Quanto à associação com as variáveis epidemiológicas estudadas, foi observada correlação positiva significativa entre idade materna e comprimento cervical, denotando tendência de associação do aumento da idade materna com maiores médias de comprimento do colo uterino. Como o estudo envolveu apenas grávidas de peso adequado, a distribuição amostral desse parâmetro não permitiu correlações com as médias de comprimento cervical uterino. Não houve diferença significativa entre pacientes brancas e não brancas, no que diz respeito às médias do comprimento do colo do útero, ao longo da gravidez.

\section{Discussão}

Numerosos estudos têm confirmado a associação do encurtamento cervical com o parto pré-termo, ainda que com ampla diferença entre os valores preditivos relatados. Revisão de 35 estudos utilizando o comprimento do colo uterino, avaliado por ultra-sonografia, para a predição do parto pré-termo, descreve variação de sensibilidade de 68 a 100\%, com especificidade variando entre 44 e $79 \%{ }^{11}$.

Por outro lado, apesar da utilidade da medida ultra-sonográfica do comprimento do colo uterino como preditor do trabalho de parto pré-termo, a sua recomendação como rotina para triagem populacional passa necessariamente pela comprovação de resultados satisfatórios obtidos com medidas terapêuticas motivadas por tais informações, caracterizando a adequação do método no que se refere à aplicabilidade clínica. Nesse sentido, a cerclagem do colo uterino, indicada por ultra-sonografia, aparece como alternativa terapêutica em muitos estudos ${ }^{12-14}$, ainda que com efetividade questionada por outros autores ${ }^{3,15}$, sen- 
do a literatura ainda carente de ensaios clínicos randomizados, com casuística representativa, que apóiem a adoção dessa conduta ${ }^{16}$.

Não obstante, é inegável que estratégia lógica para a prevenção do parto pré-termo espontâneo deva começar com a identificação das grávidas de risco, de forma que a implementação de estudos com esse objetivo permanece atual. Foram poucos os autores que se propuseram ao estudo do comportamento do comprimento cervical uterino em função das diferentes idades gestacionais, em populações não implicadas em maior risco para incompetência istmocervical ou parto pré-termo, objetivando a construção de curva de normalidade relativa a tal parâmetro.

O presente estudo difere dos antecessores, no momento em que avaliou longitudinalmente as variações do comprimento cervical uterino, no decorrer de gestações normais, construindo curva de normalidade dessa importante variável, desde o primeiro trimestre até o termo.

A observação transversal de Kushnir et al. ${ }^{8}$, utilizando a ecografia transvaginal, revelou que o comprimento do colo uterino aumenta progressivamente até a $20^{\mathrm{a}}$ ou a $25^{\mathrm{a}}$ semana gestacional, quando então começa a diminuir, sendo a velocidade de encurtamento maior entre a $31^{\text {a }}$ e a $37^{\text {a }}$ semana. Tais autores não observaram diferenças estatisticamente significativas para os resultados obtidos entre nulíparas e multíparas, à semelhança do observado na presente casuística.

Resultados análogos são descritos por Okitsu et al. ${ }^{7}$, também em estudo transversal, que compararam grávidas normais com um grupo de gestantes de risco para prematuridade e observaram associação com risco aumentado para o parto prétermo nos casos que cursaram com encurtamento cervical com velocidade superior a média e 1,5 desvios-padrão.

No estudo de Murakawa et $a 1 . .^{6}$, a análise transversal do comprimento cervical uterino entre 78 nuliparas e 99 multíparas evidenciou padrões de comportamento superponiveis para os dois grupos, ambos com redução progressiva de medidas longitudinais já a partir do segundo trimestre. Os valores médios descritos para as diferentes idades gestacionais, em ambos os grupos, foram sensivelmente inferiores aos relatados nas publicações precedentes, fato justificado pelos autores em virtude de características próprias à raça das pacientes estudadas, todas de etnia oriental.

Dois estudos longitudinais ${ }^{9,10}$ não evidenciaram mudanças significativas no comprimento cervical uterino entre a $12^{\text {a }}$ e a $31^{\text {a }}$ semana de gestação, tendo demonstrado, no entanto, importantes diferenças tanto no comprimento quanto no diâmetro cervical, entre as pacientes nuliparas e multiparas, sendo o colo das multíparas mais longo e com maior diâmetro.

Por sua vez, Iams et al. ${ }^{17}$ observaram que o colo uterino mostrou-se consideravelmente maior antes da $20^{a}$ semana de gestação, relatando encurtamento e esvaecimento graduais, sobretudo a partir da $28^{\text {a }}$ semana. O percentil 50 do comprimento cervical, na $20^{\mathrm{a}}$ semana, foi $35 \mathrm{~mm}$, tendo comprimento de $25 \mathrm{~mm}$ e $45 \mathrm{~mm}$ para os percentis 10 e 90, respectivamente. Quanto mais curto o colo, maior o risco para o parto pré-termo, sendo que esse risco duplicou quando o comprimento do colo uterino situou-se abaixo do percentil 75 (40 $\mathrm{mm}$ ) e aumentou 9,5 vezes quando o comprimento do colo esteve abaixo do percentil $10(22 \mathrm{~mm})$, por ocasião da $24^{\mathrm{a}}$ semana gestacional.

Para a amostra da população inglesa avaliada por Heath et $a .^{2}$, a média do comprimento do colo uterino na $23^{\mathrm{a}}$ semana de gestação foi de 38 $\mathrm{mm}$, sendo que em $18,8,3,4$ e $1,7 \%$ dos casos o comprimento cervical uterino foi inferior a 30,25 , 20 e $15 \mathrm{~mm}$, respectivamente.

$\mathrm{Na}$ presente amostra, para o intervalo de tempo de gravidez que compreende a $23^{\mathrm{a}}$ semana, o percentil 50 para o comprimento do colo uterino foi de $35 \mathrm{~mm}$, sendo 28 e $47 \mathrm{~mm}$ os valores correspondentes aos percentis 5 e 95, respectivamente. $\mathrm{Na}$ casuística em questão, os grupos foram equilibrados quanto ao número de nulíparas e multiparas, em ambos observando-se um encurtamento progressivo do colo uterino em função da idade gestacional. Não houve diferença significativa entre os grupos quanto às médias de comprimento cervical uterino nos intervalos de tempo avaliados, e foi possivel a construção de curva única para o comportamento do comprimento do colo uterino ao longo da gestação, com a distribuição das medidas segundo os percentis.

Nosso estudo também permitiu verificar que o intervalo entre a $20^{a}$ e a $24^{a}$ semana gestacional representa o período a partir do qual o comprimento do colo uterino encurta significativamente, em relação ao início da gravidez. Essa observação se soma aos argumentos descritos na literatura acerca da idade gestacional indicada para mensuração do colo uterino como método de triagem e rastreamento do parto pré-termo, que seria entre a $22^{\text {a }}$ e a $24^{a}$ semana de gestação ${ }^{2,18,19}$.

A abordagem das grávidas na $23^{\text {a }}$ semana gestacional reuniria ainda como argumentos favoráveis a possibilidade de realização da ultrasonografia morfológica fetal na mesma época e o fato de que a avaliação se faz numa idade gestacional na qual o produto conceptual já passa a ter condições de viabilidade, decorrido o período em que a perda fetal seria caracterizada como 
abortamento, mas ainda hábil o suficiente para a adoção de medidas terapêuticas.

Segundo Heath et al. ${ }^{2}$, a medida do colo uterino na $23^{\text {a }}$ semana de gravidez representa o melhor indicador independente para o parto antes da $32^{a}$ semana, rastreando de modo eficaz os casos de prematuridade extrema. Está demonstrado que no grupo de gestantes com comprimento cervical igual ou inferior a $15 \mathrm{~mm}$ se encontram cerca de $60 \%$ das mulheres que evoluirão com parto espontâneo até a $32^{\mathrm{a}}$ semana, $80 \%$ das que cursarão com partos até a $30^{a}$ semana e $100 \%$ daquelas cujas gestações terminarão até a $26^{a}$ semana gestacional.

$\mathrm{Na}$ população em estudo, o seguimento das gestações até o termo permitiu observar encurtamento do colo uterino mais expressivo após a $28^{a}$ semana gestacional, resultados que foram semelhantes aos relatados por outros autores ${ }^{19-21}$.

A literatura é rica em relatos a respeito da importância de características sociodemográficas no contexto da avaliação do colo uterino na predição do parto pré-termo. Nesse aspecto, os relatos de Heath et al. ${ }^{22}$ se destacam por terem objetivado especificamente tais correlações. Esses autores, avaliando o comprimento do colo do útero em 2.702 pacientes, na $23^{\text {a }}$ semana de gestação, relataram que o colo uterino mostra-se significativamente menor entre as afro-caribenhas em comparação às caucasianas, adolescentes, pacientes com baixo índice ponderal e fumantes. No planejamento do presente estudo, procurou-se excluir as gestantes que sabidamente apresentavam fatores de risco conhecidos para o parto pré-termo. Entretanto aspectos como a idade materna e raça podem ser discutidos.

Foi possivel identificar tendência de correlação do aumento da idade materna com maiores médias de comprimento do colo uterino, confirmando a idéia de que gestações em idades precoces cursam com maior risco para o parto pré-termo, como descrito na literatura ${ }^{22,23}$. Em nosso meio, estabelecer classificação precisa de amostra de pacientes quanto à raça ou etnia torna-se tarefa dificil, em reflexo à nossa grande miscigenação racial. Entre as pacientes estudadas, optou-se pela distinção entre brancas e não brancas, sendo os grupos avaliados homogêneos em relação a tais proporções, sem que houvesse diferença significativa quanto à cor materna, empregando-se análise multivariada, levando em consideração todas as variáveis comprimento cervical uterino, simultaneamente. De modo semelhante, Owen et al. ${ }^{24}$, analisando as características sociais e demográficas da população americana em relação ao parto pré-termo, não caracterizaram a raça materna como fator de risco importante.
A construção de curva de referência, fruto da observação longitudinal de gestações normais em toda sua evolução, permite a avaliação do comprimento cervical por períodos, e não apenas a adoção de um único valor de ponto de corte, numa idade gestacional restrita. A observação seriada do comprimento do colo uterino e a classificação de seu padrão de encurtamento em percentis podem ser de grande valia no seguimento das gestações de alto risco para o parto pré-termo.

É importante, contudo, reconhecer, dentro da visão multifatorial da síndrome do parto prétermo, que o emprego da ultra-sonografia transvaginal não pode ser considerado isoladamente, mas sim em concomitância a outros marcadores e estratégias de atenção pré-natal que possibilitem a identificação acurada e precoce das grávidas de maior risco para o parto pré-termo e, conseqüentemente, a instituição oportuna das medidas terapêuticas, viabilizando a redução dos indices de morbidade e mortalidade perinatais.

\section{ABSTRACT}

Purpose: to establish a normality curve of cervical length during pregnancy measured by transvaginal ultrasonography.

Methods: we conducted a prospective, longitudinal study on 82 healthy pregnant women who were followed up from the beginning of pregnancy to delivery at four-week intervals, of whom 49 concluded the study. Patients were divided according to parity into nulliparous women and women with one or more previous deliveries. Cervical length was measured in a sagittal view by transvaginal ultrasonography, as the linear distance between internal and external cervical os.

Results: no significant difference was observed in mean cervical length or the 5th, 25, 50th, 75th, or 95th percentile according to gestational age between groups $(p>0.05)$. Between the 20th and 24th gestacional week, the 5th. 50th and 95th percentiles of cervical length were 28, 35 and 47.2 $\mathrm{mm}$, respectively. Cervical length decreased progressively during normal pregnancy, with a significant shortening observed after 20 weeks of gestation and being more marked after 28 weeks $(p<0.05)$.

Conclusions: the pattern of cervical length behavior does not seem to differ between nulliparous women and women with one or more previous deliveries. The numerical values of the normality curve of cervical length according to gestational age reflect the variability in the peculiar characteristics of the studied sample, thus emphasizing the value of the parameters established for different populations.

KEYWORDS: Cervical length. Normal pregnancy. Transvaginal ultrasonography. 


\section{Referências}

1. Mozurkewich EL, Naglie G, Krahn MD, Hayashi RH. Predicting preterm birth: a cost-effectiveness analysis. Am J Obstet Gynecol 2000; 182:1589-98.

2. Heath VC, Southall TR, Souka AP, Elisseou A, Nicolaides KH. Cervical length at 23 weeks of gestation: prediction of spontaneous preterm delivery. Ultrasound Obstet Gynecol 1998; 12:312-7.

3. Berghella V, Daly SF, Tolosa JE, et al. Prediction of preterm delivery with transvaginal ultrasonography of the cervix in patients with high-risk pregnancies: does cerclage prevent prematurity? Am J Obstet Gynecol 1999; 181:809-15.

4. Hassan SS, Romero R, Berry SM, et al. Patients with an ultrasonographic cervical length $\leq 15 \mathrm{~mm}$ have nearly a $50 \%$ risk of early spontaneous preterm delivery. Am J Obstet Gynecol 2000; 182:1458-67.

5. Andersen HF, Nugent CE, Wanty SD, Hayashi RH. Prediction of risk for preterm delivery by ultrasonographic measurement of cervical length. Am J Obstet Gynecol 1990; 163:859-67.

6. Murakawa H, Utumi T, Hasegawa I, Tanaka K, Fuzimori R. Evaluation of threatened preterm delivery by transvaginal ultrasonographic measurement of cervical length. Obstet Gynecol 1993; 82:829-32.

7. Okitsu O, Mimura T, Nakayama T, Aono T. Early prediction of preterm delivery by transvaginal ultrasonography. Ultrasound Obstet Gynecol 1992; 2:402-9.

8. Kushnir O, Vigil DA, Izquierdo L, Schiff M, Curet LB. Vaginal ultrasonographic assessment of cervical length changes during normal pregnancy. Am J Obstet Gynecol 1990; 162:991-3.

9. Zorzoli A, Soliani A, Perra M, Caravelli E, Galimberti A, Nicolini U. Cervical changes throughout pregnancy as assessed by transvaginal sonography. Obstet Gynecol 1994; 84:960-4.

10.Cook CM, Ellwood DA. A longitudinal study of the cervix in pregnancy using transvaginal ultrasound. Br J Obstet Gynaecol 1996; 103:16-8.

11.Leitich H, Brunbauer M, Kaider A, Egarter C, Husslein P. Cervical length and dilatation of the internal cervical of detected by vaginal ultrasonography as markers for preterm delivery: a systematic review. Am J Obstet Gynecol 1999; 181:1465-72.

12. Heath VCF, Souka AP, Erasmus I, Gibb DM, Nicolaides KH. Cervical length at 23 weeks of gestation: the value of Shirodkar suture for the short cervix. Ultrasound Obstet Gynecol 1998; 12:318-22.
13.Guzman ER, Forster JK, Vintzileos AM, Ananth CV, Walters C, Gipson K. Pregnancy outcomes in women treated with elective versus ultrasoundindicated cervical cerclage. Ultrasound Obstet Gynecol 1998; 12:323-7.

14.Althuisius SM, Dekker GA, Hummel P, Bekedam DJ, van Geijn HP. Final results of the Cervical Incompetence Prevention Randomized Cerclage Trial (CIPRACT): therapeutic cerclage with bed rest versus bed rest alone. Am J Obstet Gynecol 2001; 185:1106-12.

15.Rust OA, Atlas RO, Reed J, van Gaalen J, Balducci J. Revisiting the short cervix detected by transvaginal ultrasound in the second trimester: why cerclage therapy may not help? Am J Obstet Gynecol 2001; 185:1098-105.

16.ACOG Practice Bulletin. Assessment of risk factors for preterm birth. Clinical management guidelines for obstetrician-gynecologists. Number 31, October 2001. Obstet Gynecol 2001; 98:709-16.

17.Iams JD, Goldenberg RL, Meis PJ, et al. The length of the cervix and the risk of spontaneous premature delivery. N Engl J Med 1996; 334:567-72.

18. Oliveira TA, Carvalho CMP, et al. Avaliação do risco de parto prematuro: teste da fibronectina fetal e medida do colo uterino. Rev Bras Ginecol Obstet 2000; 22:633-9.

19. Carvalho MHB, Bittar RE, Gonzales M, Brizot ML, Zugaib M. Avaliação do risco para parto prematuro espontâneo pelo comprimento do colo uterino no primeiro e segundo trimestres da gravidez. Rev Bras Ginecol Obstet 2002; 24:463-68.

20.Danti L, Palai N, Ravelli V, Lojacono A, Tanzi P, Bianchi UA. Studio ecografico del collo dell'utero in gravidanza. Curva di normalità in uno studio longitudinale e transversale. Minerva Ginecol 1998; 50:397-404.

21.Gramellini D, Fieni S, Molina E, Berretta R, Vadora E. Transvaginal sonographic cervical length changes during normal pregnancy. J Ultrasound Med 2002; 21:227-32.

22. Heath VC, Southall TR, Souka AP, Novakov A, Nicolaides KH. Cervical length at 23 weeks of gestation: relation to demographic characteristics and previous obstetric history. Ultrasound Obstet Gynecol 1998; 12:304-11.

23. Creasy RK, Gummer BA, Liggins GC. System for predicting spontaneous preterm birth. Obstet Gynecol 1980; 55:692-5.

24. Owen J, Goldenberg RL, Davis RO, Kirk KA, Copper RL. Evaluation of a risk scoring system as a predictor of preterm birth in an indigent population. Am J Obstet Gynecol 1990; 163:873-9. 